\title{
Erythritol. Functionality in noncaloric functional beverages*
}

\author{
Peter de Cock $^{\ddagger}$ and Claire-Lise Bechert \\ Cerestar, Vilvoorde R\&D Centre, Havenstraat 84, 1800 Vilvoorde, Belgium
}

\begin{abstract}
Noncaloric functional beverages are a key segment in the rapidly growing functional food market. Erythritol, an all-natural bulk sweetener, offers sensorial as well as functional benefits for use in such beverages. It can improve taste quality by adding body and mouthfeel, and it is capable of masking unwanted off-flavors often associated with intense sweeteners. In addition, erythritol can strengthen the functional concept since it is noncaloric, noncariogenic, and nonglycemic, and it has antioxidant properties.
\end{abstract}

\section{INTRODUCTION}

The beverage market has in recent years seen drastic changes as consumers are increasingly longing for a healthier lifestyle and well-being. In our modern society, stress, fatigue, and loss of vitality are just a few examples of this century's so-called "western disease". Consequently, beverage manufacturers' interest turned to a market that has existed now for quite some time in Japan, the functional drink market. Functional drinks are indeed a logical response to consumers' expectations, and popular concepts include boosting the immunity system, relieving stress, improving intestinal health, increasing vitality and stamina, controlling cholesterol and body weight, and fighting degenerative diseases.

The variety of ingredients used - as well as the health and nutrition claims-are rapidly growing. The functional beverage market covers many categories, from sports/energy drinks to herbal teas and fruit and vegetable juices. Specific groups are targeted: seniors, adults, teenagers, children, and even toddlers. For all of these consumer groups, there is one golden rule to be considered by product developers: No one is willing to sacrifice taste to health and nutrition benefits.

Erythritol is an all-natural moderately sweet bulk sweetener (60 to $70 \%$ as sweet as sucrose) with taste and mouthfeel enhancing properties. It naturally occurs in various fruits and fermented foods and is produced by a natural fermentation process. Erythritol is well suited for functional beverages because it is, among others, noncaloric and nonglycemic, and it acts as an antioxidant. This ingredient helps formulators ensure a good-tasting quality of their new developments as well as provide significant health benefits.

\section{GLOBAL FUNCTIONAL MARKET DEVELOPMENTS}

The global functional food market reached a value of $\$ 31.7$ billion in 1999 with an expected annual growth of over $10 \%$ until 2004 [1]. The market now is led by the United States, followed by Europe and Japan. The area of development for these various world regions is closely linked to their cultural eating habits. The focus is on bakery products in America and on probiotic dairy products in Japan. In Europe, where cultural differences have to be considered on a country basis, the emphasis for functional

*Pure Appl. Chem. Vol. 74, No. 7, 2002. A special topic issue on the science of sweeteners.

ॠCorresponding author 
developments for the main three markets in the United Kingdom, France, and Germany is on breakfast cereals, dairy products, and beverages, respectively.

Today, however, the trend has clearly changed, as shown by the number of new product launches in the food and drink sector. The beverage category has significantly increased worldwide and is seen as the best vehicle for functional ingredients.

The functional ingredients and health claims for new developments can also differ from one world area to another. In fact, in the United States, after the era of "light" and "diet" products, consumers are now moving to more "natural" concepts. The use of botanical and herbal extracts such as gingko, echinacea, etc. and of vitamins and minerals in beverages is very popular. Anticancer ingredients are also becoming increasingly important. In Japan, the prevention of potential age-related health issues such as coronary diseases, vitality loss, and bone weakness are the main focus. In a country that has the oldest population in the world, the main target logically is the elderly group. In parallel, an increased interest in "cosmetic" and "beauty" foods appears on the market, with products claiming to improve skin or hair condition. In Europe, the focus on digestive benefits is predominant. Prebiotics such as inulin, fructooligosaccharides, fibers, and resistant starch easily find their way into successful new products.

Even though differences exist on a global basis, with functional drinks, beverage manufacturers unanimously found a way to develop tailor-made products for every segment of the population. The new beverage concepts are meant to answer health concerns specific to the different age groups and genders. For the elderly population, fruit juices with calming properties are being developed as well as beverages that help prevent or cure heart diseases. For adults, the focus is on weight and cholesterol management, tension reduction, and improvement of the metabolism and libido. Beverages specifically designed for pregnant or post-menopausal women and for women's inner balance and harmony are also available. For the younger population, mental fitness and concentration while studying are crucial. Single-portion still or carbonated drinks supplemented with gingko, ginseng, or guarana have become quite popular. Also, following the American pattern, unhealthy eating habits and, consequently, increasing obesity have arisen in Europe and Asia. Vitamin- and mineral- fortified fruit juices, and pre- and probiotic light chocolate drinks are helping teens and children manage this issue.

Finally, even the little ones are being considered. Beverages in the form of fortified liquid meal replacements, powdered drinks, and fruit juices are designed for better growth, increased mental alertness and performance, as well as improved rehydration of toddlers.

Functional beverages on the market encompass a large variety of products. From energy and sports drinks and teas of all sorts (green, black, oolong, and herbal), to juice drinks, dairy drinks, and smoothies, the choice is infinite. Even some alcoholic beverages have a functional image in Japan. But whatever the beverage category targeted for new product development, the success relies on a few specific criteria. The main and indisputable one is taste. Consumers will never sacrifice taste to health properties.

Secondly, consumers' expectations in terms of end-products really depend on the health benefit being claimed. For example, in Europe, an energy drink is expected to have a thin texture, while a calming beverage should be slightly thicker with a higher juice content. Cultural differences across the world could lead to different expectations.

And last, but not least, health benefits should always be substantiated with scientific data. In this growing functional drink market, the lack of regulation and the absence of technical substantiation could simply end up in the collapse of the market. Misleading or false statements could result in the mistrust from consumers. To prevent this from happening, in Japan, the Food for Specific Health Use (FOSHU) approval was implemented by the government back in 1991. It enabled consumers to feel confident in the claims being made. However, in the rest of the world, formal regulations hardly exist, and, therefore, beverage manufacturers should try to comply with the above criteria in order to maintain credibility. 


\section{Improving taste}

As previously stressed, taste is critical to obtaining a successful drink. Currently on the market, traditional low-calorie beverages, as opposed to the so-called "new age" drinks, remain very different from their sugar-based counterparts. They lack the taste characteristics and mouthfeel provided by sucrose. Thus, when these low- or noncaloric drinks were first introduced, subgroups were formed among consumers. The population was divided into "regular users", loyal to the sucrose taste, and "diet users", who found in artificially sweetened drinks a hedonically satisfactory alternative. With the introduction of functional drinks, in their regular or noncaloric versions, this division will likely continue. Moreover, the potential off-tastes exhibited not only by intense sweeteners but also by certain functional ingredients will need to be overcome to win consumer acceptance.

Erythritol is used today by many beverage manufacturers. It has a very clean sweet taste profile similar to sucrose, and its bulk properties provide a significant mouth feel in solution, as shown in Fig. 1. More importantly, these taste benefits translate into finished beverages.

Figure 2 shows the results of a taste panel, where erythritol was added to an increasingly popular functional drink, i.e., tea. A significant increase of mouthfeel and decrease in astringency and bitterness

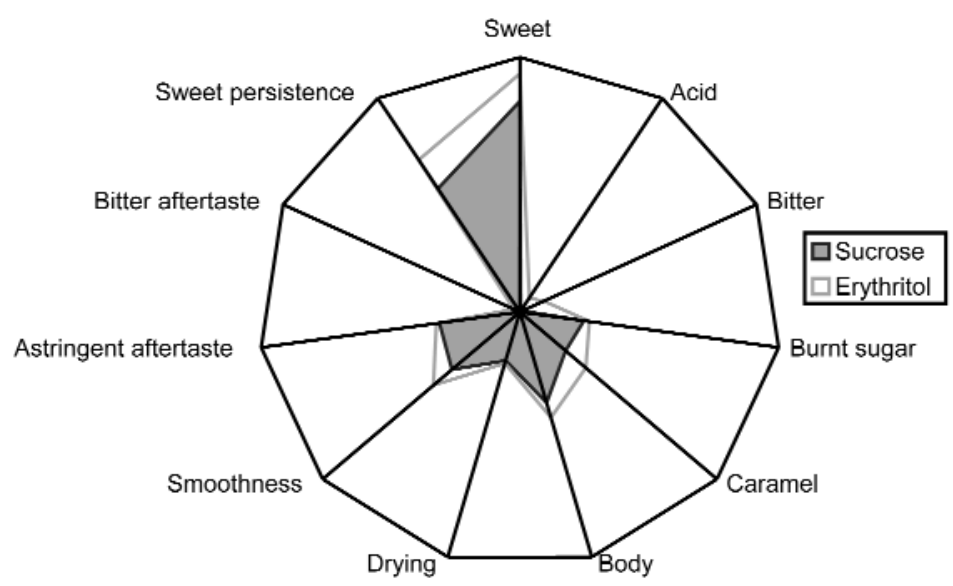

Fig. 1 Taste profile of erythritol and sucrose in water at $10 \%$ sucrose equivalent value (SEV).

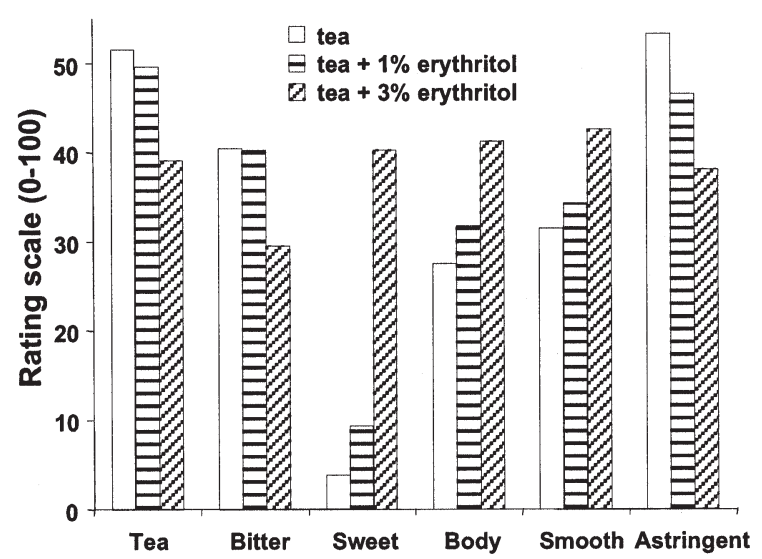

Fig. 2 Effects of erythritol added at 1 and $3 \%$ on the flavor profile of tea. 
was observed upon adding $3 \%$ erythritol to tea. This is why erythritol, when used alone in noncaloric beverages, gains popularity even with the most conservative regular sucrose users.

If used in combination with intense sweeteners, erythritol shows an improvement of the taste quality of the beverage. In combination with sucralose at $1.5 \%$, or at $3 \%$ with aspartame, acesulfame-K or saccharin, erythritol provides significant mouthfeel and masks potentially unpleasant characteristics exhibited by intense sweeteners or functional ingredients. Figure 3 shows the positive taste impact of $1.5 \%$ erythritol on a sucralose solution. Erythritol increases the smoothness and body mouthfeel, as well as the natural caramel after-taste, and decreases astringent, irritant, and cooling characteristics.

Not only does erythritol provide qualitative synergy in blend with intense sweeteners, it also exhibits a quantitative sweetness synergy, as represented in Table 1 . When erythritol is the major contributor to the sweetness, additive synergy is obtained in combination with aspartame. The maximum synergy of $30 \%$ is obtained for an 85:15 erythritol:aspartame blend ratio. Additive synergy is also observed in blends with acesulfame-K. Only minute amounts of these high-intensity sweeteners are necessary to boost the sweetness intensity of erythritol by about $30 \%$.

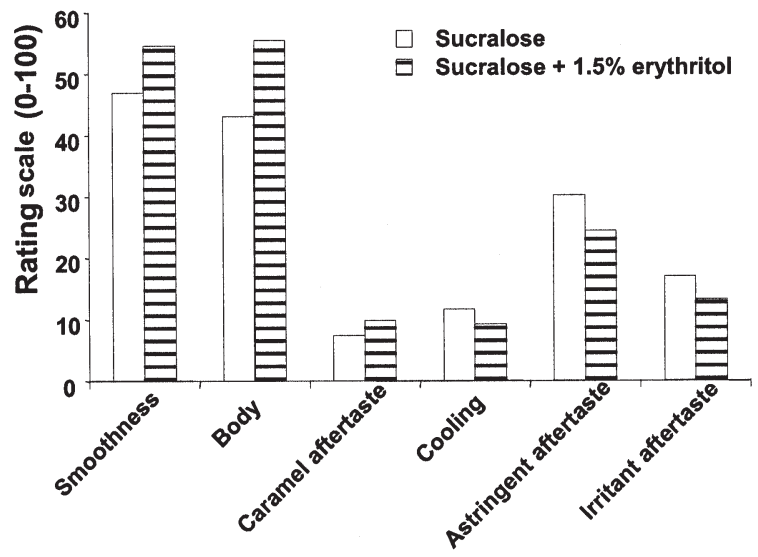

Fig. 3 Taste profile of sucralose and sucralose-erythritol blend in water at $10 \%$ SEV.

Table 1 Quantitative sweetness synergy (\%) of erythritol/aspartame and erythritol/acesulfame-K blends. Expected sweetness of $10 \%$ SEV for all blends.

\begin{tabular}{lcccccc}
\hline & \multicolumn{7}{c}{ Erythritol-intense sweetener ratio (sweetness contribution) } \\
Sweetener combinations & $1-99$ & $5-95$ & $15-85$ & $85-15$ & $95-5$ & $99-1$ \\
\hline Erythritol/aspartame blend & -3 & -7 & 10 & $30^{\mathrm{a}}$ & $25^{\mathrm{a}}$ & $24^{\mathrm{a}}$ \\
Erythritol acesulfame-K blend & 12 & 8 & $19^{\mathrm{a}}$ & $32^{\mathrm{a}}$ & $31^{\mathrm{a}}$ & $27^{\mathrm{a}}$ \\
\hline
\end{tabular}

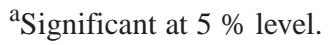

\section{Taste improvement of low-calorie functional beverage upon storage}

Erythritol exhibits an important stability under acidic and processing conditions used in beverage manufacturing and upon storage. It remains stable under prolonged exposure to a $\mathrm{pH}$ ranging from 2 to 10 . Erythritol is also heat-stable at temperatures up to $160{ }^{\circ} \mathrm{C}$. Furthermore, erythritol does not undergo Maillard browning reaction. Thanks to this great stability, erythritol's significant contribution to improving the taste of freshly made artificially sweetened beverages in fresh products persists-in fact increases-upon storage. 
A nine-month shelf-life experiment was performed in a noncaloric carbonated orange drink. Two references were used, one sweetened with aspartame and one with an aspartame/acesulfame-K blend. These were compared to test samples containing aspartame plus an increasing concentration of erythritol $(0.5 \%$ up to $3 \%)$. In the fresh products, the qualitative synergy previously discussed was confirmed by a trained sensory panel.

Upon storage, the aspartame slowly degraded, as shown in Table 2, leading to a decrease in the sweetness intensity of the orange drinks. Thus, the overall acceptability of the drinks decreased in the reference samples. Erythritol (partly) compensates for the loss of sweetness encountered with aspartame over time. It also masks potential off-flavors developing upon storage. Consequently, erythritol improves the overall taste quality of aspartame-sweetened drinks and extends their shelf life.

Table 2 Sweetener concentration upon storage for aspartame, acesulfame-K, and erythritol.

\begin{tabular}{lccc}
\hline & \multicolumn{3}{c}{ Remaining level of sweetener $(\%)$} \\
& 3 months & 6 months & 9 months \\
\hline Erythritol & 100 & 100 & 100 \\
Acesulfame-K & 100 & 100 & 100 \\
Aspartame & $70-72$ & $52-56$ & $46-47$ \\
\hline
\end{tabular}

\section{Erythritol strengthens the desired image of functional beverages}

Erythritol not only improves taste quality in functional beverages. It also has a number of nutrition and health benefits that are high in demand in the functional food arena.

\section{Noncaloric characteristics of erythritol}

One of erythritol's unique characteristics is the fact that it is noncaloric $(\leq 0.2 \mathrm{kcal} / \mathrm{g})$, and therefore very useful as a bulk sweetener in weight-management programs. There is no other bulk sweetener with such a low caloric value, owing to erythritol's unique metabolic profile. Erythritol's low molecular weight allows more than $90 \%$ of its ingested form to be readily absorbed by passive diffusion from the small intestine. Since the human body does not have any enzymes that can break down erythritol, it is not metabolized and is excreted unchanged in the urine. The remaining fraction of $<10 \%$ reaches the large intestine and may be fermented by the colonic microflora, resulting in the production of volatile fatty acids (VFAs), biomass, and gases $\left(\mathrm{H}_{2}, \mathrm{CH}_{4}\right)$. The VFAs are absorbed and metabolized in the liver, thereby making about half of the intrinsic caloric value of the carbohydrate available to the body [2]. Consequently, the overall caloric value of erythritol ranges from 0 to a maximum of $0.2 \mathrm{kcal} / \mathrm{g}$.

\section{Glycemic or insulinemic response}

Clinical studies demonstrate that consumption of erythritol does not raise plasma glucose or insulin levels. This makes erythritol a suitable sweetener for people who suffer from diabetes. In a study done by Bornet et al. [3], mean plasma glucose and insulin levels, measured for up to $3 \mathrm{~h}$ after ingestion of a single dose of $1 \mathrm{~g}$ erythritol/kg body wt, were unaffected by erythritol (see Fig. 4).

The importance of low-glycemic foods is gaining more and more attention owing to their potential benefits. These include a lower risk of developing Type 2 diabetes; a lower probable risk of a hypoglycemic episode, long-term diabetic complications, and coronary heart disease; and assistance in managing obesity. The importance of the glycemic index was the subject of a recent international workshop entitled Glycemic Index and Health: The Quality of the Evidence [4]. The overall conclusion of the 48 experts attending the workshop was that current evidence indicates that the glycemic index has practical utility and that differences in the glycemic index among foods may have important relevance to public health. They also recommended further basic and epidemiological research and controlled clinical 

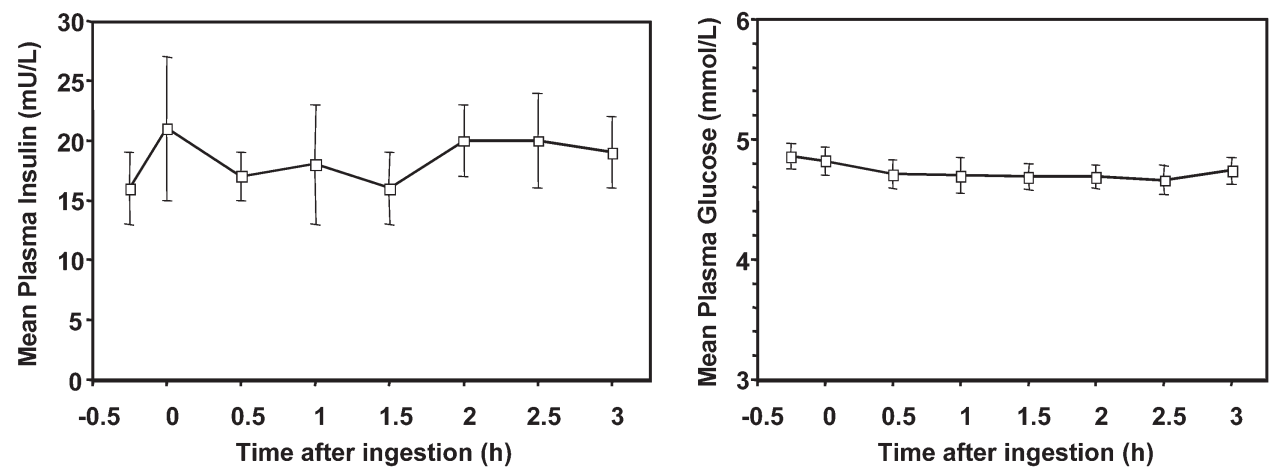

Fig. 4 Mean plasma glucose and insulin concentration after a $1 \mathrm{~g} / \mathrm{kg}$ body weight single oral intake of erythritol by six healthy human volunteers. Error bars indicate standard error of the mean (SEM).

trials in the area of weight management, prevention and management of cardiovascular disease, diabetes and cancer, and cognitive function.

\section{Highest digestive tolerance of all polyols}

It is well established that the consumption of excessive amounts of polyols can provoke undesirable intestinal side effects such as laxation, abdominal cramps, flatulence, and-in extreme cases-watery diarrhea. Some of these symptoms are the result of osmotic effects, and others are the result of the fermentative degradation of these compounds in the colon. Numerous human tolerance studies have shown that the incidence and severity of these intestinal side effects and their threshold dose depend upon the particular polyol consumed, the mode of ingestion, the existence of a previous adaptation period, and the individual susceptibility for this kind of effect.

Although erythritol chemically belongs to the group of polyols, it has a digestive tolerance that is much higher compared to all other polyols. Since $>90 \%$ of the ingested erythritol is readily absorbed from the small intestine, minimal amounts reach the lower gut. Consequently, under the anticipated conditions of use, erythritol does not cause laxation. Clinical studies from Bornet et al. [3] and Tetzloff et al. [5] show that gastrointestinal effects in adults ingesting erythritol at up to $1 \mathrm{~g} / \mathrm{kg}$ body wt (up to $80 \mathrm{~g} /$ day) were not statistically different from those in persons ingesting sucrose at similar levels.

Erythritol's high digestive tolerance is particularly advantageous when used in beverages since the intake from beverages can be relatively high. A use level of 1 to $3 \%$ is usually sufficient to generate the sensorial benefits described earlier in this article. A concentration of $3.5 \%$ erythritol is isotonic.

Table 3 lists the maximum bolus dose not causing laxation of a number of polyols. These figures were generated in clinical studies that were conducted under most severe conditions: nonadapted test

Table 3 Maximum bolus dose not causing laxation of erythritol and other polyols.

\begin{tabular}{lccc}
\hline Polyol & \multicolumn{2}{c}{$\begin{array}{c}\text { Maximum bolus dose not causing laxation } \\
(\mathrm{g} / \mathrm{kg} \text { body wt) }\end{array}$} & Reference \\
\cline { 2 - 3 } & Male & Female & \\
\hline Erythritol & 0.66 & 0.80 & {$[6]$} \\
Sorbitol & 0.17 & 0.24 & {$[6]$} \\
Maltitol & 0.3 & 0.3 & {$[8]$} \\
Isomalt & 0.3 & - & {$[7]$} \\
Xylitol & 0.3 & 0.3 & {$[9]$} \\
\hline
\end{tabular}


subjects consumed a single bolus dose as a liquid or jelly on an empty stomach within $10 \mathrm{~min}$. Such test conditions allow for better tolerance comparison and simulate worst intake conditions from beverages.

In a very recent clinical study [10], the digestive tolerance of erythritol was compared to xylitol under similar severe conditions (single bolus dose as a liquid on an empty stomach). In this doubleblind, controlled cross-over study, 65 young adults consumed $400 \mathrm{ml}$ of orange-flavored drinks containing either $0,20,35$, or $50 \mathrm{~g}$ of xylitol or erythritol (45 g sucrose was used as control). Results on laxation are shown in Fig. 5. This study clearly demonstrates that consumption of 35 and $50 \mathrm{~g}$ xylitol is associated with significant increases in the frequency and magnitude of gastrointestinal responses, including severe laxation compared to equivalent doses of erythritol or $45 \mathrm{~g}$ sucrose.

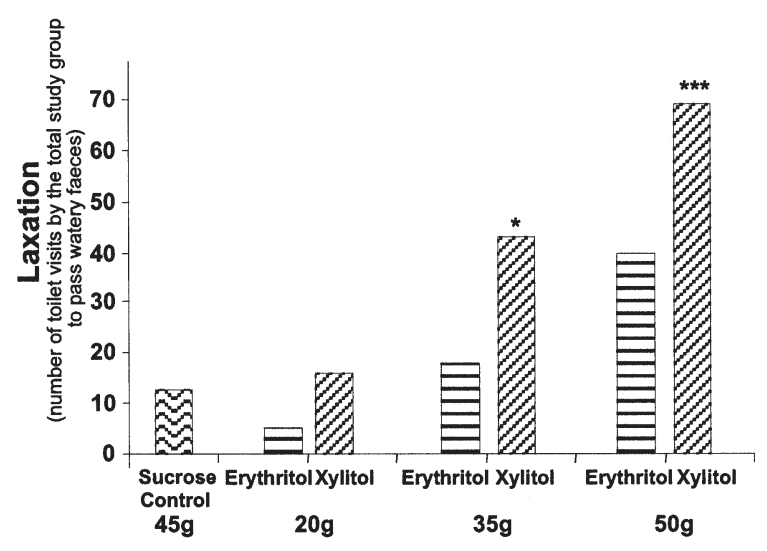

Fig. 5 Results on laxation. Significant difference from sucrose at $* 5 \%$, ***0.5 $\%$ level.

\section{Noncariogenicity of erythritol}

The noncariogenicity of erythritol has been demonstrated using various methods. In vitro incubation with a range of Streptococci species has shown that neither lactic acid [11] nor other organic acids are produced from erythritol. Under the same test conditions, xylitol gave a similar response, whereas maltitol, sorbitol, mannitol, lactitol, and isomalt showed minor acid production.

Streptococci were also unable to grow on erythritol and, therefore, could not produce the glucosyltransferase that enables them to synthesize their insoluble glucan plaque material. This was confirmed in separate in vitro tests showing that Streptococci did not produce any polymeric plaque material and, subsequently, could not adhere to a glass surface when incubated on erythritol, in contrast to the control groups incubated on sucrose.

The in vivo plaque $\mathrm{pH}$ test is commonly accepted as the reference method for measurement of the cariogenic potential of foods. This test is based on the formation of organic acids in dental plaque after exposure to fermentable dietary carbohydrates and measures the corresponding decrease of plaque $\mathrm{pH}$. The potential of a food to cause low plaque $\mathrm{pH}$ values is generally agreed to be closely associated with the initiation of dental decay [12]. If the plaque $\mathrm{pH}$ value after ingestion of a food remains above the critical $\mathrm{pH}$ value of 5.7, the food is regarded as safe for teeth. Erythritol was studied in a plaque $\mathrm{pH}$ test in the form of a crystallized tablet (lozenge). An example of a $\mathrm{pH}$ curve from the intraplaque measurements during chewing of erythritol tablets is represented in Fig. 6. Sucrose is used as a control. All tests consistently demonstrated the noncariogenicity of erythritol.

\section{Hydroxyl radical scavenger}

Formation of highly reactive hydroxyl radicals $\left(\mathrm{OH}^{\circ}\right)$ is catalyzed by metals such as iron and copper. The hydroxyl radical reacts at a very high rate with virtually any biomolecule by hydroxylation, hydro- 


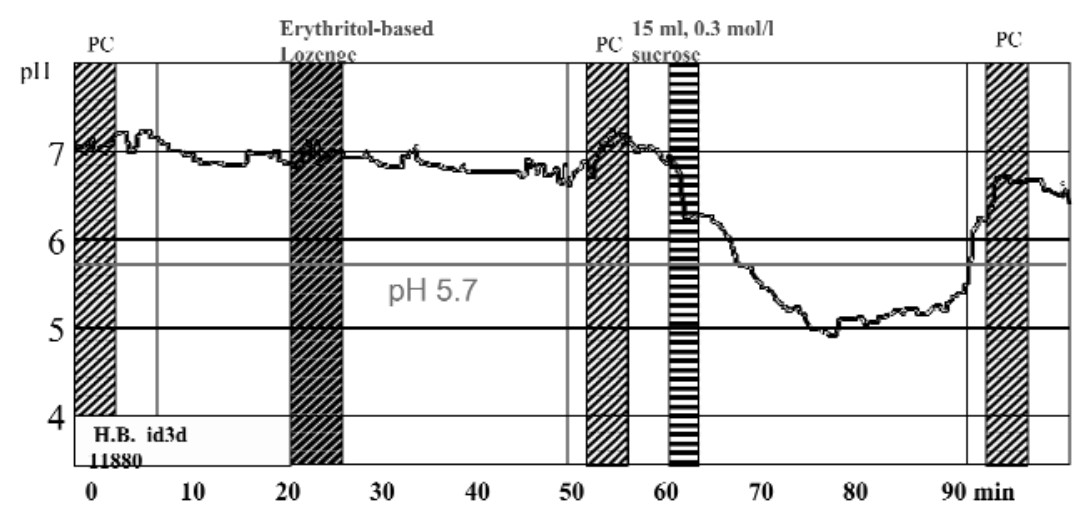

Fig. 6 Example of a plaque pH curve of interdental plaque in one volunteer during and for 30 min after consumption of an erythritol-based lozenge. A $0.3 \mathrm{~mol} / \mathrm{l}(10 \%)$ sucrose rinse for $2 \mathrm{~min}$ was used as positive control. $(\mathrm{PC})=$ paraffin chewing. $(\mathrm{id})=$ age of interdental plaque $(\mathrm{l})$ in days $(\mathrm{d})$.

gen abstraction, or oxidation. This results in damaged proteins, membranes, and DNA, eventually causing cellular dysfunction or cell death. Biomolecules can be protected against radicals by radical scavengers or antioxidants [13]. Scavengers are compounds that react with the radical before the radical reacts with the biomolecule. This prevents the damage of proteins, membranes, and DNA and, therefore, protects the cell against harmful effects of radicals.

It has been reported that polyols such as mannitol possess antioxidant properties [14]. In a recent in vitro study [15], hydroxyl radical scavenging activity of erythritol and other polyols (sorbitol, xylitol, and mannitol) was confirmed. Since erythritol, as opposed to other polyols, is so well absorbed for $\geq 90 \%$ and not metabolized, it has a great potential to act as an efficient hydroxyl radical scavenger in the human body. Further research is underway to confirm and quantify this potential functional benefit.

Many beverage producers and consumers have already recognized the sensorial and functional benefits of erythritol. Table 4 summarizes some of the most popular functional beverage concepts and the potential benefits of using erythritol in such concepts.

Table 4 Benefits of erythritol in popular functional beverage concepts.

\begin{tabular}{lll}
\hline Functional concept & \multicolumn{1}{c}{ Targeted health condition } & \multicolumn{1}{c}{ Erythritol benefit } \\
\hline "Beauty from inside" & Anti-aging & Antioxidant \\
& Fight caries & Noncariogenic \\
& Body weight control & Noncaloric \\
"Senior health" & Cholesterol control & No specific effect \\
& Glycemic control & Nonglycemic \\
& Body defense against degeneration/inflammation & Free-radical scavenger \\
& Antistress & No specific effect \\
& Digestive comfort & High tolerance \\
& Body weight control & Noncaloric \\
\hline
\end{tabular}

\section{Global regulatory status of erythritol}

The safety of erythritol is well documented $[16,17]$. On the basis of its large safety database, erythritol is expanding approval for use around the world with the list of countries growing all the time. At present, Japan, the United States, Australia, New Zealand, Taiwan, Singapore, Korea, and, recently, Russia, Israel, and South Africa have approved its use in foods. Petitions are pending in many other countries, 
including the European Union, Canada, Mercosur, and China. Erythritol also has been awarded the valuable international endorsement of Joint FAO/WHO Expert Committee on Food Additives (JECFA), and is now included in the General Standard for Food Additives (GSFA-list) of the Codex Alimentarius under INS number 968. Alongside these regulatory approval expansions, we are also seeing a widening range of consumer products successfully using erythritol; these developments are supported in the United States by an extension of generally recognized as safe (GRAS) uses and use levels. The recent GRAS notification by the Food and Drug Administration (FDA) reaffirms the safety of erythritol and its wide interest in the food and beverage industry.

\section{CONCLUSION}

Erythritol is an all-natural bulk sweetener with unique sensorial and functional properties. Alone or in combination with intense sweeteners, erythritol improves the flavor and mouthfeel characteristics of low-calorie and diet beverages throughout their shelf life. Since erythritol has a high digestive tolerance and is noncaloric, noncariogenic, nonglycemic, and has antioxidant properties, it fits well functional drink concepts to which it confers a healthy image. Erythritol therefore enables product developers to design great-tasting and truly new functional beverages.

\section{REFERENCES}

1. Euromonitor. Functional Foods: A World Survey (2000).

2. A. J. H. van Es. "Energy utilisation of low digestibility carbohydrates", in D. C. Leegwater et al. (Eds.), pp. 121-127, Wageningen, Pudoc (1987).

3. F. Bornet, A. Blayo, F. Dauchy, G. Slama. Regul. Toxicol. Pharmacol. 24, S296-S302 (1996).

4. Danone Vitapole. Workshop organized by Danone Vitapole and LU, with the technical contribution of the Food and Agriculture Organisation of the United Nations, 21-23 February, Bandol, France (2001).

5. W. Tetzloff, F. Dauchy, S. Medimagh, D. Carr, A. Bar. Regulat. Toxicol. Pharmacol. 24, S286-S295 (1996).

6. T. Oku and M. Okazaki. Nutrition Res. 16 (4), 577-589 (1996).

7. T. Oku. Nutrition Rev. 54 (11), S59-S66 (1996).

8. N. Koizumi, M. Fujii, R. Ninomiya, Y. Inoue, T. Kagawa, T. Tsukamoto. Chemosphere 12, 45-53 (1983).

9. BIO Clinica. "Estimated maximum dose not causing laxation of various sweeteners and their reference", reported (in Japanese) in BIO Clinica 11 (11), (817) 31 (1996).

10. D. Storey and A. Lee. "Study to evaluate the comparative digestive tolerance of young adults following consumption of drinks containing either erythritol or xylitol" (2001). Unpublished.

11. J. Kawanabe, M. Hirasawa, T. Takeuchi, T. Oda, T. Ikeda. Caries Res. 26, 258-362 (1992).

12. Scientific consensus conference on methods for assessment of the cariogenic potential of foods, San Antonio, Texas, 17-21 November. J. Dental Res. 65, 1473-1543 (1985).

13. A. Bast, G. Haenen, C. Doelman. Am. J. Med. 91, 2S-13S (1991).

14. B. Halliwell, J. Gutteridge, O. Aruoma. Anal. Biochem. 165, 215-219 (1987).

15. G. den Hartog, G. Haenen, A. Bast. "Superoxide and hydroxyl radical scavenging activity of erythritol and other polyols" (2001). Unpublished.

16. I. C. Munro, W. O. Berndt, J. F. Borzelleca, W. G. Flamm, B. S. Lynch, E. Kennepohl, A. Bär, J. Modderman. Food Chem. Toxicol. 36, 1139-74 (1998).

17. WHO. "Erythritol", in Safety Evaluation of Certain Food Additives and Contaminants, WHO Food Additives Series, 44, pp. 15-70, ICPS International Program on Chemical Safety in Cooperation with the Joint FAO/WHO Expert Committee on Food Additives (JECFA), World Health Organisation, Geneva (2000). 